\title{
Kernos
}

Revue internationale et pluridisciplinaire de religion grecque antique

19 | 2006

Varia

\section{La dernière génération héroïque}

Un parcours historico-religieux et sémio-narratif, d'Hésiode au ps.Apollodore

\section{Giovanni Tosetti}

\section{(2) OpenEdition}

\section{Journals}

Édition électronique

URL : https://journals.openedition.org/kernos/440

DOI : $10.4000 /$ kernos.440

ISSN : 2034-7871

\section{Éditeur}

Centre international d'étude de la religion grecque antique

\section{Édition imprimée}

Date de publication : 1 janvier 2006

Pagination : 113-130

ISSN : 0776-3824

\section{Référence électronique}

Giovanni Tosetti, «La dernière génération héroïque », Kernos [En ligne], 19 | 2006, mis en ligne le 20 mars 2011, consulté le 24 août 2022. URL : http://journals.openedition.org/kernos/440 ; DOI : https:// doi.org/10.4000/kernos.440 


\title{
La dernière génération héroïque : un parcours historico-religieux et sémio-narratif, d'Hésiode au ps.-Apollodore
}

\begin{abstract}
Résumé : La présente étude considère les héros qui participèrent à la guerre de Troie en tant que génération située à la fin des successions généalogiques chez Hésiode, chez quelques logographes - tels qu'Acousilaos d'Argos, Hécatée de Milet, Hellanicos de Lesbos et Phérécyde d'Athènes - et le Ps.-Apollodore de la Bibliothèque. Au moyen d'une méthode à la fois historicoreligieuse et sémio-narrative, on se propose de montrer comment, chez ces auteurs, la génération de la guerre de Troie et celle des épigones, qui suit immédiatement, concluent souvent les récits généalogiques, en marquant la fin du temps des héros, après lequel s'ouvre un régime temporel différent, celui du bic et nunc.
\end{abstract}

\begin{abstract}
The Last Heroes' Generation: A Historico-religious and Semio-narrative Survey from Hesiod to Ps.-Apollodorus. The present study concerns Trojan War heroes as the final generation in the genealogical stories composed by Hesiod, by some logographers - such as Hekataios of Miletos, Akousilaos of Argos, Pherekydes of Athens and Hellanikos of Lesbos - and by the Ps.Apollodorus of the Library. By means of both a historico-religious and semio-narrative method, we aim to demonstrate that, according to the work of these authors, the Trojan generation and the epigonoi, who follow immediately afterwards, often conclude the genealogical stories. This marks the end of the heroic age, after which begins a different time, that of the bic et nunc.
\end{abstract}

\section{Objet et méthode}

L'Hymne pseudo-homérique à Aphrodite ${ }^{1}$ introduira la présente étude, avec laquelle on aimerait considérer la dernière génération du temps héroï$q^{2} e^{2}$ chez Hésiode, quelques généalogistes en prose ${ }^{3}$ comme Hécatée, Acousilaos, Phérécyde et Hellanicos, et, enfin, le Ps.-Apollodore de la Bibliothèque.

\footnotetext{
${ }^{1}$ Pour une introduction au corpus des ainsi dits Hymnes bomériques, cf. CASSOLA (19997), p. IX-LXXVI. En ce qui concerne en particulier le rapport entre ces poèmes et leurs circonstances d'énonciation, $c f$. CALAME (1996a), p. 485-492. - Pour une présentation synthétique du débat scientifique qui concerne la datation de l'Hymne à Aphrodite, cf. CAssola (19997), p. 247-251, qui pense que l'Hymne remonte au VII ${ }^{e}$ s. av. J.-C., mais n'exclut pas une chronologie postérieure. JANKO (1982), p. 151, sur des bases linguistiques, soutient que l'Hymne à Aphrodite a été composé dans l'Éolie asiatique du Nord après la Théogonie d'Hésiode, mais avant l'Hymne à Déméter. Il pourrait dater de l'époque d'Hésiode, à savoir, d'après Janko, de la première moitié du VII ${ }^{e}$ s. D'autres savants, tel que HoEkSTRA (1969), p. 39-40, pensent à la deuxième moitié du $\mathrm{VII}^{\mathrm{e}} \mathrm{s}$.

2 Au moyen de cet énoncé académique, nous nous référons à la période à laquelle vécurent les mortels que, comme on le verra, Hésiode, Travaux 159-160, identifie avec la « divine génération des hommes héros, lesquels sont nommés demi-dieux ».

${ }^{3}$ Cette dénomination est assez fréquente : cf. ThOmas (1989), p. 173; JACOB (1994), p. 177.
} 
En effet, le récit hymnique ${ }^{4}$ de l'amour entre Aphrodite et Anchise met en scène un point essentiel d'articulation du temps des héros, puisque c'est à ce moment-là que s'accomplit la dernière union divino-humaine, après laquelle il n'y aura plus ni d'intimité amoureuse entre immortels et mortels, ni, naturellement, de naissances semi-divines 5 . Zeus, en effet, pour mettre fin aux amours divino-humains causés par Aphrodite, inspire à la déesse même une passion amoureuse envers un mortel, Anchise. Aphrodite, en éprouvant la douleur qui saisit un immortel à la suite de l'amour avec un être humain et la naissance d'un fils mortel, ne pourra plus provoquer de telles unions? .

Sur la base de ces remarques il nous a semblé convenable de considérer le traitement, dans la tradition des récits généalogiques, de la fin de l'âge hérö̈que par rapport à la guerre troyenne. C'est, en effet, lors de ce conflit qu'Énée, le fils de la dernière union divino-humaine, ainsi que les héros contemporains accomplirent leurs exploits.

Au moyen d'une méthode historico-religieuse, nous chercherons à vérifier si tout au long de notre parcours diachronique à travers différentes formes discursives on peut identifier, dans la vision indigène de la fin de l'âge héroïque, des analogies ${ }^{8}$ à savoir la présence de comparabilités fondées aussi bien sur des similarités que sur des différences.

Les sources que nous avons mentionnées seront étudiées au moyen d'une analyse du niveau discursif, tout en visant à identifier aussi bien les indications temporelles qui concernent les articulations du temps héroïque que les énonciations du bic et nunc?

\section{Corpus hésiodique}

\subsection{Catalogue final de la Théogonie}

Nous aimerions commencer notre enquête par le catalogue final de la Théogonie ${ }^{10}$ hésiodique, puisque il dresse d'une manière très claire et synthé-

${ }^{4}$ Cf. Hymne à Aphrodite (V), 45-52 et 196-200.

$C f$. les observations très claires de CLAY (1989), p. 166-170.

${ }^{6}$ Hymne à Aphrodite (V), 45-52

7 Ibid., 247-255.

${ }^{8}$ Dans la méthode historico-religieuse de U. Bianchi et des savants qui relèvent de sa démarche, la notion d'«analogie » est tout à fait essentielle, puisque elle permet de valoriser aussi bien les similarités que les différences entre les phénomènes religieux, dont on cherche à vérifier une certaine homogénéité tout en reconnaissant et en interprétant les diversités. Cf. Bianchi (1979), p. 16, 57-59, 80-83, 104-105, 116, 146-147 et 159.

9 Sur l'analyse sémio-narrative, $c f$. CALAME (1996²), p. 29-60, (1996b), p. 46-55, et (2000), p. $47-52$.

${ }^{10}$ Hésiode, Théogonie, 963-1018. En ce qui concerne l'établissement du texte, nous nous bornons ici à observer que dans ce passage le vers 1014 est incertain, puisque il pourrait être interpolé : $c f$. WEST (1966), p. 434-435. Il est bien connu que la majeure partie des savants pense que la Théogonie originelle se terminait avant la conclusion du poème tel qu'il nous est parvenu, même s'il y a différentes hypothèses sur l'extension de la partie finale qui serait interpolée : $c f$. WEST (1966), p. 398-399, qui croit que la nouvelle conclusion aurait été introduite, au $\mathrm{VI}^{\mathrm{e}}$ s. av. J.- 
tique une liste diachronique ${ }^{11}$ d'unions entre déesses et mortels et de leurs fils semi-divins.

Les vers ${ }^{12}$ qui nous intéressent le plus concernent les amours dont naissent des héros qui participèrent à la guerre de Troie $^{13}$ :

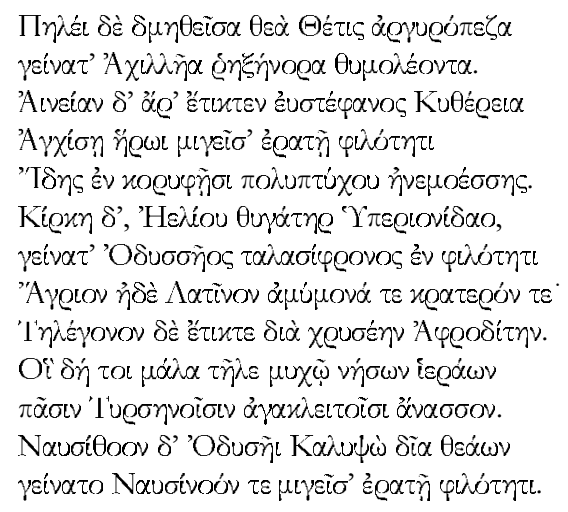

La présentation d'Achille ${ }^{14}$ et d'Énée ${ }^{15}$ se fonde sur un chiasme pour en souligner la comparabilité selon le schéma A-B-C C-B-A, où A est le nom du père au datif, $\mathrm{B}$ le nom de la mère au nominatif et $\mathrm{C}$ le nom du fils à l'accusatif. Les vers suivants ${ }^{16}$, eux-mêmes en disposition de chiasme, mentionnent Ulysse en tant que mortel aimé, respectivement, par Circé et Calypso, qui ont chacune engendré des fils. Ainsi ce catalogue d'unions divino-humaines se termine-t-il par une référence aussi bien à la guerre de Troie, avec Achille et Énée, qu'à la phase suivante des retours, représentée par Ulysse, dont sont mentionnés les fils comme emblèmes de ce qu'on pourrait dénommer la génération des épigones. Le temps raconté ${ }^{17}$ de la

C., par le rhapsode même du Catalogue des femmes. Les arguments de West n'ont pas convaincu tous les érudits : $c f$. ARrighETTI (1998), p. 368-371; DräGER (1997), p. 1-26, qui pense que la conclusion de la Théogonie, comme le Catalogue des femmes, doit être attribuée à Hésiode.

${ }^{11}$ Cf. CLAY (2003), p. 164.

${ }^{12}$ Hés., Théogonie, 1006-1018

13 «La déesse Thétis au pied d'argent soumise à Pélée engendra Achille qui détruit les troupes, au coeur de lion. Cythérée à la belle couronne enfantait Énée, en s'étant unie d'amour désirable au héros Anchise sur les sommets de l'Ida riche de replis et venteux. Circé, fille d'Hélios fils d'Hypérion, engendra de son union avec le patient Ulysse Agrios et Latinos, irréprochable et fort; elle enfantait Télégonos à cause d'Aphrodite d'or. Ils régnaient très loin à l'intérieur d'îles sacrées sur tous les illustres Tyrrhéniens. Calypso, divine parmi les déesses, engendra Nausithoos et Nausinoos à Ulysse, en s'étant unie d'amour désirable. »

${ }^{14}$ Ibid., 1006-1007.

15 Ibid., 1008-1010

16 Ibid., 1011-1018

${ }^{17}$ En parlant de «temps raconté », nous désignons évidemment le temps qui voit se dérouler les événements racontés, à savoir, dans le cas présent, le temps des héros. Pour de récentes réflexions sur le temps raconté, aussi bien que d'autres distinctions temporelles «intra- » et « extra-discursives », $c f$. CALAME (1998), p. 342-343, et (1999), p. 66-67 et 78-83. 
Théogonie, donc à travers un déroulement diachronique, aboutit aux générations humaines des guerriers de Troie, représentés aussi bien par les demi-dieux Achille et Énée que par Ulysse, qui sont suivis par le genos né des participants au conflit troyen. Autour de la guerre de Troie, la dernière période héroïque présente donc des articulations internes, en enrichissant le témoignage constitué par le récit de l'Hymne à Aphrodite.

\subsection{Le genos héroïque dans le récit des cinq espèces humaines}

Dans la célèbre narration que les Travaux et les Jours ${ }^{18}$ consacrent aux cinq âges humains, l'espèce dans laquelle s'inscrit le locuteur ${ }^{19}$, à savoir le genos de fer, est immédiatement précédée par la «divine génération des hommes héros, lesquels sont nommés demi-dieux $»^{20}$. Il s'agit des héros qui participèrent aux conflits de Thèbes et de Troie, et y moururent ${ }^{21}$. Ils sont dits demidieux parce qu'un certain nombre d'entre eux est né d'un amour divinohumain et les autres comptent, parmi leurs ancêtres, un dieu ou une déesse.

La dénomination de «demi-dieux» est ancrée dans le bic et nunc de l'énonciation, comme le montre l'emploi du présent de l'indicatif $\varkappa \alpha \lambda \varepsilon ́ o v \tau \alpha \iota$, et renvoie à un intérêt généalogique pour les liens de parenté entre immortels et mortels. En effet, les savants ont souvent souligné comment les poèmes homériques et hésiodiques attestent, en de nombreux passages, que les familles aristocratiques du temps de l'énonciation se réclamaient de la descendance des héros et donc, en dernier lieu, de quelque divinitée $e^{22}$.

Tandis qu'entre les quatre premières espèces humaines il y a une coupure nette, car, après la disparition de celle qui a existé avant, la suivante est produite soit par les Olympiens ${ }^{23}$ soit, en particulier, par Zeus ${ }^{24}$, le passage de l'âge des héros à la période actuelle ne semble pas comporter une séparation complète par rapport au passé. Le locuteur, en effet, ne mentionne pas la fabrication de l'espèce de $\mathrm{fer}^{25}$. Si, en plus, on considère que, par rapport à la

\footnotetext{
${ }^{18}$ Hés., Travaux, 106-201.

19 Ibid., 174-175.

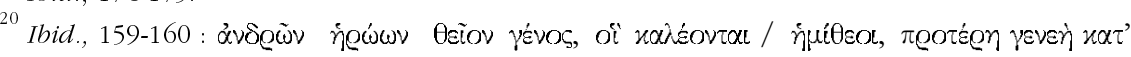

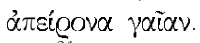

${ }^{21}$ Ibid. 162-165.

22 On se bornera ici à renvoyer à WEST (1985), p. 8-9; LÉVÊQUE (1985), p. 186-187, 200 et 211; MURRAY (1996), p. 175

23 Les Olympiens sont en général mentionnés comme les auteurs des espèces d'or et d'argent : Hés., Travaux, 109-110, 127-128.

${ }^{24}$ Zeus produit la génération de bronze et celle hérö̈que : Hés., Travaux, 143-144, 157-158.

${ }^{25}$ Cf. maintenant CALAME (2004b), p. 84. Il faut, toutefois, remarquer que les savants ne sont pas tous d'accord sur cette hypothèse, car on objecte qu'il faut distinguer « entre la narration et les événements qu'elle expose » : l'absence d'une référence à la fabrication de l'humanité de fer serait tout simplement un silence narratif qui ne signifierait pas que l'événement ne s'est pas passé (Crubellier [1996], p. 439-440). Il nous semble intéressant, néanmoins, d'observer que, dans le hic et nunc de l'énonciation de poèmes généalogiques tels que le Catalogue des femmes, l'intérêt pour les genè héroïques dépend du fait que les familles aristocratiques contemporaines réclamaient leur descendance des héros (cf. supra n. 22). Il semble dès lors douteux qu'Hésiode
} 
génération actuelle, le locuteur emploie le mot geneé, au lieu de genos, auquel il recourt tout au long du récit pour désigner les espèces humaines, il est possible d'interpréter cette variation lexicale comme une indication généalogique au sens restreint: l'humanité hérö̈que à travers la succession des générations serait en rapport de continuité avec les hommes de l'âge de fer ${ }^{26}$. Il nous semble donc possible de conclure que le présent récit montre comment l'âge héroïque, caractérisé par l'humanité tout à fait particulière des demi-dieux, s'est terminé, tout en gardant, néanmoins, des liens généalogiques avec les hommes du «maintenant» de l'énonciation, lesquels, en descendant des $\dot{r} \mu i ́ \theta \varepsilon o \iota$, peuvent rattacher en dernier lieu leurs lignées à une divinité.

Cet étroit lien généalogique entre l'espèce humaine des héros et celle de fer, mis en valeur par les familles situées dans le bic et nunc, peut expliquer la collocation, souvent considérée comme problématique ${ }^{27}$, du quatrième genos immédiatement avant la génération humaine du temps de l'énonciation du poème.

\subsection{Hés., fr. 204 M.-W.}

Le Catalogue des femmes ${ }^{28}$ est un poème consacré exclusivement aux générations mortelles issues des amours entre dieux et femmes. Le temps raconté correspond donc à la période dont l'Hymne à Aphrodite identifie la fin avec l'union de la déesse et Anchise et dont s'occupent les ouvres hésiodiques considérées: la Théogonie en dressant un catalogue final d'unions divino-humaines, les Travaux en se concentrant sur la nature, la fin et la survivance des héros dits demi-dieux.

Comme le locuteur de l'Hymne, celui du Catalogue met en rapport, dans le fr. 204 M.-W., la fin des demi-dieux avec un dessein de Zeus. Il s'agit d'une tradition qui, avec similarités et différences sur lesquelles on ne peut pas ici se concentrer, est clairement attestée, en ce qui concerne l'épique préclassique, par le prélude des Cypria ${ }^{29}$ et, peut-être, par celui de l'Iliade ${ }^{30}$.

n'ait pas considéré cette tradition qui était bien enracinée dans la culture grecque d'époque préclassique. Il n'a donc probablement pas parlé de fabrication de l'humanité de fer parce qu'il concevait celle-ci comme descendant de la génération héroïque.

${ }^{26}$ Pour ce signifié de geneé, $c f$. maintenant CALAME (2004b), p. 81

${ }^{27}$ En effet, on a pensé que l'espèce hérö̈que interrompait la succession, dans une ligne de décadence, des quatre autres âges humains. Pour une récente référence critique aux perspectives savantes qui, au Xx s., ont été consacrées à ce récit, $c f$. CALAME (2004b), p. 69-71.

${ }^{28}$ Il est bien connu que le problème de la paternité et, donc, de la datation du Catalogue des femmes demeure ouvert. L'hypothèse que le poème n'est pas hésiodique et a été composé au VI s. av. J.-C., dans la période 580-520, a été proposée avec succès par WeST (1985), p. 130-137. Parmi les savants qui ont accepté la proposition de West, en n'attribuant pas le poème à Hésiode, on se bornera ici à nommer MARCH (1987), p. 157-159, qui a restreint l'arc temporel, en proposant une datation entre 580 et 570, et HIRSCHBERGER (2004), p. 42-51, qui date le Catalogue entre 630 et 590. D'autres savants affirment que le poème est hésiodique. Il suffira, ici, de renvoyer aux travaux de MERKELBACH (1968), p. 126-133, et, récemment, DrÄGER (1997), p. 1-16.

${ }^{29}$ Fr. 1 (éd. BERNABÉ). 
Dans des vers de lecture très difficile ${ }^{31}$, il semble que le locuteur montre

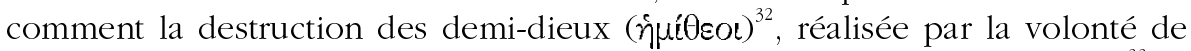
Zeus, est finalisée, si on accueille une intégration proposée par A. Rzach ${ }^{33}$, à un rétablissement d'une nette distinction ${ }^{34}$ entre la sphère immortelle et celle mortelle. Les personnages nés des unions divino-humaines donc se révèlent problématiques pour l'ordre cosmique, qui, pour le futur comme pour un passé précédant cette période d'intimité, devra se fonder sur une coupure nette entre plan divin et humain. Le dessein de Zeus prépare donc cette distinction, présente et reconnue comme nécessaire dans le nunc de l'énonciation.

La destruction des demi-dieux semble s'accomplir au moyen de la guerre de Troie $^{35}$, comme le laisse penser la place de ces vers après la mention des prétendants d'Hélène ${ }^{36}$.

Le Catalogue des femmes confirmerait donc que la guerre de Troie a causé la fin de l'humanité héroïque.

\section{Les logographes}

\subsection{Acousilaos d'Argos}

Entre la fin de la période archaïque et le début de l'époque classique, par l'intermédiaire des généalogistes qu'on nomme souvent logographes ${ }^{37}$, des œuvres en prose commencent à être consacrées aux générations divines et surtout hérö̈ques.

Déjà dans l'antiquité, l'un des auteurs de ces suggraphai ${ }^{38}$, Acousilaos d'Argos ${ }^{39}$, a été rapproché d'Hésiode ${ }^{40}$. Ce jugement nous semble pertinent car

30 I, 1-7. Cf. récemment Rousseau (2001), p. 151-152, qui a montré, à travers une analyse détaillée de la narration iliadique, que les auditeurs étaient stimulés à observer, au fil du récit, l'accomplissement d'un plan de Zeus qui, différemment des Chants Cypriens, n'était pas prémédité par rapport à la guerre troyenne, mais, en même temps, dans une relation dialectique avec les événements du conflit, conduisait, comme dans le poème cyclique, l'humanité héroïque à son terme.

${ }^{31}$ Hés., fr. 204, 95-104 M.-W.

32 Ibid., 100.

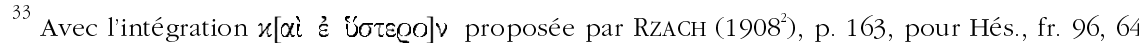

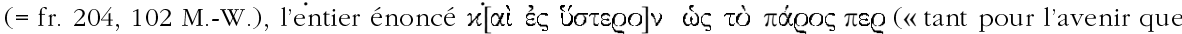
le passé ») exprimerait que la nouvelle et définitive distinction entre immortels et mortels reprend une séparation qui existait déjà avant l'époque des unions divino-humaines. Sur cette articulation interne au temps raconté du Catalogue, cf. CERUTTI (1998), p. 174-177.

${ }^{34}$ Hes., fr. 204, 103 M.-W.

35 Cf. WeST (1985), p. 119-121; récemment, CERUTTI (1998), p. 146.

${ }^{36}$ Hés., fr. 204, 41-95 M.-W.

37 Sur l'emploi de ce terme, $c f$. VON FRITZ (1967), p. 79 et 337-347 (« Excurs I »).

${ }^{38}$ Pour l'emploi indigène de ce terme, $c f$. CALAME (2004a), p. 230-235.

39 Sur le floruit de ce logographe, les savants sont d'accord : cf. récemment FowLER (2000), p. 1, qui le date ante bellum Persicum et LendLe (1992), p. 10, qui colloque le sommet de sa carrière autour du 500 av. J.-C. 
ses Généalogies reprennent la structure du corpus hésiodique, en commençant par les phases théogoniques ${ }^{41}$ de la constitution du cosmos et poursuivant par les généalogies des héros, auxquelles est dédiée la majeure partie de l'œuvre. D'après nous, on peut affirmer que dans les Généalogies le temps des héros se terminait, de la même manière que dans les poèmes de la tradition hésiodique, avec la guerre troyenne et les événements qui lui sont étroitement liés. Cette hypothèse se fonde sur trois fragments qui mentionnent le livre dont ils sont issus. Le premier est un témoignage lexicographique $e^{42}$ qui atteste que, dans le dernier des trois livres dont se composaient les Généalogies, Acousilaos affirmait, à l'instar d'Hellanicos, que les Homérides sont ainsi nommés à partir du nom d'Homère. Comme l'a signalé $\mathrm{F}$. Jacoby ${ }^{43}$, il est probable que cette information au sujet d'Homère était présentée dans la section sur la guerre de Troie. On peut donc conclure que ce conflit était traité à la fin des Généalogies.

Une scolie iliadique ${ }^{44}$ cite un autre passage d'Acousilaos où est présentée la généalogie d'Échépolos, un prince de Sicyone qui a obtenu de ne pas aller à Troie en faisant, à la place, un don à Agamemnon. Le scoliaste place ce passage au dernier livre des Généalogies, en confirmant que les événements troyens étaient racontés à la fin de l'œuvre.

La mention du retour d'Ulysse appartenait sans doute aussi au dernier livre, puisque une scolie aux Argonautiques d'Apollonios ${ }^{45}$ atteste que, dans cette partie de l'œuvre, Acousilaos présentait la naissance des Phéaciens à partir des gouttes d'Ouranos tombées à terre après sa castration par Cronos. Or, comme les Phéaciens sont déjà dans l'Odyssée ${ }^{46}$ associés au retour d'Ulysse, il est probable qu'Acousilaos les a mentionnés dans ce contexte ${ }^{47}$.

On peut donc penser que le logographe terminait ses Généalogies avec la guerre de Troie suivie par les retours ${ }^{48}$, selon un déroulement du temps

${ }^{40}$ Ménandre Rhéteur, 338, 5-9 = Acousilaos, test. 4 (éd. Fowler) $=2 \mathrm{~T} 4$ (éd. JACOBY); Clement d'Alexandrie, Stromates VI, 26, 8 = Acous., test. 5 (éd. Fowler) $=2 \mathrm{~T} 5$ (éd. JACOBY) Joseph, Contre Apion I, 16 = Acous., test. 6 (éd. Fowler) = 2 T 6 (éd. JACOBy). Cf. JACOB (1994), p. 174-176; BERTELli (2001), p. 73; CALAME (2004a), p. 233-235.

41 Acousilaos commençait ses généalogies théogoniques avec les mêmes archai de la Théogonie hésiodique : cf. Platon, Banquet, 178ab = Acous., fr. 6a (éd. Fowler) = 2 F 6a (éd. JACOBY) et Damascius, Traité des premiers principes, $124^{1}$, avec les observations de CALAME (2004a), p. 235-236.

${ }^{42}$ Harpocration, XV, 19 (222, 10 [éd. Dindorf], 191 [éd. KEANey]) = Acous., fr. 2 (éd. Fowler) = 2 F 2 (éd. JACOBY)

43 (1957²b), p. 376 ad 2 F 2.

${ }^{4}$ Schol. (T) Homère, Iliade XXIII, 296 = Acous., fr. 3 (éd. Fowler) = 2 F 3 (éd. JACOBY).

45 Schol. (L+) Apollonius de Rhodes, IV, 982-921 = Acous., fr. 4 (éd. Fowler) $=2$ F 4 (éd. JACOBY).

${ }^{46} \mathrm{~V}, 388$; XIII, 187.

47 Cf. JACOBY $\left(1957^{2}\right.$ b), p. 376 ad 2 F 4

48 JACOBY $\left(1957^{2}\right.$ b), p. 376 ad 2 F 1-4, observe que les premiers quatre fragments des Généalogies attestent que la syggraphè commençait avec les unions théogoniques et se terminait avec les événements troyens et les retours. 
héroïque analogue à celui qui est attesté dans le catalogue final de la Théogonie.

Sur la base de ces trois témoignages, il est donc possible d'attribuer au troisième livre les autres fragments d'Acousilaos qui, sans indication de la partie de l'œuvre, concernent les événements troyens ${ }^{49}$.

En particulier, il nous semble intéressant de considérer une scolie iliadi$q^{50}$ qui nous présente la cause du conflit troyen telle qu'elle était identifiée dans les Généalogies. Acousilaos, en reprenant une tradition à laquelle le rhapsode iliadique faisait seulement allusion ${ }^{51}$, développe une explication de la guerre qui relève de son intérêt généalogique. En effet, il raconte qu'Aphrodite a engendré Énée de son union amoureuse avec Anchise et a poussé Alexandre à enlever Hélène pour permettre l'accomplissement d'un oracle, selon lequel, après le renversement de la lignée de Priam, aurait régné sur les Troyens celle d'Anchise. En donnant naissance au fils d'Anchise et en causant la guerre de Troie sans, ensuite, aider véritablement les troupes troyennes à éviter la défaite, elle aurait favorisé la chute du règne de Priam et préparé le pouvoir d'Énée et de ses descendants. La réflexion d'Acousilaos sur la guerre de Troie ne semble donc pas se concentrer sur une perspective intéressée, comme dans le corpus hésiodique et, surtout, dans le Catalogue des femmes, à la distinction entre le plan des immortels et celui des mortels, mais sur un point de vue strictement généalogique et de pouvoir royal. Les origines de la guerre de Troie sont identifiées par Acousilaos avec des stratégies généalogiques de souveraineté sans aucune référence au problème de l'intimité divinohumaine, comme c'était le cas, d'une manière très claire, avec le fragment 204 de Merkelbach-West.

\subsection{Hécatée de Milet}

Au sujet du fameux logographe presque contemporain d'Acousilaos, Hécatée de Milet $^{52}$, on ne possède pas de fragments consacrés explicitement à la guerre de Troie. On observera, toutefois, qu'il est possible que cet auteur ait traité le conflit, puisque, dans le dernier livre de ses Généalogies, il s'est occupé de l'Asie Mineure, en faisant supposer un intérêt pour la fameuse guerre qui a glorifié cette région ${ }^{53}$. De plus, on a avancé l'hypothèse ${ }^{54}$ qu'une

${ }^{49}$ Fr. 39-43 (éd. FOWLER) = 2 F 39-43 (éd. JACOBY).

50 Schol. Hom., Iliade XX, $307=$ Acous., fr. 39a (éd. Fowler) = 2 F 39 (éd. JAcoby).

${ }^{51}$ Hom., Iliade XX, 306-308.

52 FOwLER (2000), p. 110, le date avant la rébellion ionienne de 499-494 av. J.-C.; LENDLE (1992), p. 10 et 18, tout en situant sa naissance autour de 550 av. J.-C., précise qu'Acousilaos était un contemporain un petit peu plus jeune que lui.

53 Cette hypothèse a déjà été proposée par JACOBY (1957²b), p. 320-321 ad 1 F 10-11.

${ }^{54}$ Cf. JACOBY $\left(1957^{2} \mathrm{~b}\right)$, p. 321 ad $1 \mathrm{~F} 11$. Comme me l'a suggéré R.L. Fowler dans une conversation orale, on ne peut pas, toutefois, exclure qu'Hécatée mentionne Mélie en référence à la migration grecque en Ionie. En effet, on verra que cette colonisation, considérée comme postérieure à la guerre de Troie, était connue par Phérécyde et Hellanicos : $c f$. SAKELLARIOU (1958), p. 307-308 
référence à Mélie ${ }^{55}$, une ville de la Carie, provient d'une partie consacrée aux nostoi, car la Bibliothèque du Pseudo-Apollodore ${ }^{56}$ nous informe que Podaleirios, après la guerre de Troie, s'établit dans la Chersonèse de la Carie. On peut donc penser que, comme dans la partie finale de la Théogonie et chez Acousilaos, chez Hécatée aussi le temps héroïque raconté à travers une diachronie généalogique se terminait avec la guerre de Troie suivie par les retours.

\subsection{Phérécyde d'Athènes}

Phérécyde d'Athènes ${ }^{57}$ consacrait également une partie de ses Histoires aux événements de Troie et aux retours ${ }^{58}$, mais l'absence de toute indication nous empêche de savoir dans lequel des dix livres se déroulait ce récit ${ }^{59}$. En tout cas, un passage de Strabon ${ }^{60}$ témoigne du fait que ce généalogiste racontait un événement postérieur aux Troica, à savoir la migration des Ioniens en Asie Mineure, région au sujet de laquelle Phérécyde mentionnait aussi la phase pré-ionienne ${ }^{61}$.

Pour ce qui est d'une diachronie relative, il est possible d'observer que lui même racontait, après la guerre troyenne, les nostoi. En effet, il se réfère à la monstrueuse Scylla affrontée par Ulysse lors de son retour de Troie et précise quels compagnons du héros moururent face à ce danger ${ }^{62}$, en confirmant l'importance particulière, dans la tradition sur les retours, du voyage d'Ulysse ${ }^{63}$.

Une autre importante indication de chronologie relative concerne la place de la guerre de Thèbes par rapport au conflit troyen. En effet, ce logographe ${ }^{64}$ témoigne très bien de la manière dont ce conflit entre Argiens et Thébains précède immédiatement la guerre de Troie. On peut donc affirmer que les

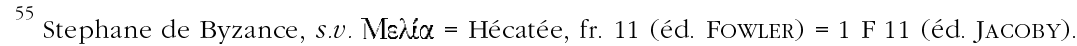

${ }^{56}$ Ps.-Apollodore, Bibliothèque, épit. VI, 18.

57 Pour des datations récentes de son activité, $c f$. FOwLER (2000), p. 272, qui propose l'âge de Cimon, et LENDle (1992), p. 18 et 22, qui, plus en général, indique la première moitié du ve siècle av. J.-C.

${ }^{58}$ Fr. 136-144 (éd. FOWLER) = 3 F 136-144 (éd. JACOBY).

59 JACOBY (1957a), p. 95, propose, dubitanter, que ce sujet était présenté dans le livre IX, l'avant-dernier de l'œuvre.

${ }^{60}$ Strabon, XIV, 1, 3 = Phérécyde, fr. 155 (éd. FOWLER) = 3 F 155 (éd. JACOBY). Sur ce sujet, $c f$. SAKELlariou (1958), p. 307; JACOBY (1957²b), p. 426-427 ad 3 F 155; récemment, LuRAGHI (2000), p. 362-366.

${ }^{61}$ À Strab., XIV, 1, 3 = Phéréc., fr. 155 (éd. FOwLER) = 3 F 155 (éd. JACOBY), on ajoutera, en ce qui concerne la fondation de Téôs par Athamas d'Orchomène avant l'arrivée des Ioniens, Schol. (T+) Pl., Hipparque, 229d = Phéréc., fr. 102 (éd. Fowler) = 3 F 102 (éd. Jacoby); $c f$. JacoBy (1957²b), p. 418 ad 3 F 102.

${ }^{62}$ Schol. $(\mathrm{HX}+)$ Hom., Odyssée XII, 257 = Phéréc., fr. 144 (éd. Fowler) = 3 F 144 (éd. JACOBY).

${ }^{63}$ On se bornera ici à rappeler que déjà dans Hom., Odyssée I, 11-15, Ulysse est présenté comme le héros qui n'était pas encore rentré chez lui, quand les autres Grecs survivants avaient désormais accompli leur retour.

${ }^{64}$ Schol. (T) Hom., Iliade XIII, 663 = Phéréc., fr. 115 (éd. FOwler) = 3 F 115a (éd. JACOBY)
} 
événements troyens suivaient ceux de Thèbes, d'après une tradition qui, attestée dès Homère, ${ }^{65}$ était racontée dans les Cypria ${ }^{66}$, et, comme on l'a vu, était présentée d'une façon très claire dans le récit hésiodique des cinq âges humains.

En ce qui concerne la structure des Histoires, le rapport de Phérécyde avec la tradition hésiodique semble, toutefois, moins étroit que celui entretenu avec Hésiode par Acousilaos. En effet, il n'est pas sûr que l'œuvre de Phérécyde commençait, comme celle du généalogiste argien, par la phase théogonique, si bien formalisée par la Théogonie ${ }^{67}$.

En outre, déjà dans le premier livre était mentionné l'amour de Pélée et Thétis, qui se situe dans les dernières phases du temps héroïque, comme en témoigne bien le catalogue final de la Théogonie. Le choix de placer cette union au début de la syggraphé s'explique par l'intention de commencer la narration par le genos des Éacides, dont descendait la famille des Philaides, très importante dans le hic et nunc de l'énonciation, comme l'atteste un fragment bien connu de Phérécyde ${ }^{68}$ sur lequel on ne peut pas s'arrêter ici.

Il suffira de remarquer qu'on est ici en présence du premier témoignage clair d'une généalogie qui, sans solution de continuité et génération après génération, relie le temps des héros à un passé récent très significatif pour la situation d'énonciation. Elle se déroule, en effet, de Philée, fils d'Ajax, le fameux héros né de Télamon, jusqu'à Miltiade, le fondateur de la Chersonèse et grand-père du vainqueur de Marathon ${ }^{69}$. Si déjà dans les Travaux le locuteur semblait vouloir remarquer une continuité généalogique entre le genos hérö̈que et le genos de fer, le lien est davantage encore souligné ici en énonçant, de génération en génération, le déroulement ininterrompu du lignage.

Ainsi, chez Phérécyde, l'attention au développement interne de chaque lignée héroïque l'emporte sur un intérêt, à l'œuvre chez Hésiode, pour la formation graduelle du cosmos avec ses articulations entre phase théogonique, période héroïque et fin de cette dernière en tant qu'intimité particulière entre les dieux et les hommes.

\footnotetext{
${ }^{65}$ Hom., Iliade IV, 370-400 (première guerre de Thèbes, qui surgit de la rivalité entre Étéocle et Polynice); ibid. 404-410 (deuxième guerre de Thèbes, à laquelle participèrent Diomède et les autres epigonoi); ibid. V, 800-808 (première guerre de Thèbes); ibid. VI, 222-223 (première guerre de Thèbes); ibid. X, 285-286 (première guerre de Thèbes); ibid. XIV, 113-114 (première guerre de Thèbes); Odyssée XV, 246-247 (première guerre de Thèbes).

${ }^{66}$ Fr. 1, test. I-IV (éd. Bernabé).

${ }^{67}$ VON Fritz (1967), p. 83, nie cette possibilité avec décision, alors que FowLer (2000), p. 302-303, ne l'exclut pas totalement.

${ }^{68}$ Marcellinus, Vie de Thucydide II-IV = Phéréc., fr. 2 (éd. Fowler) = 3 F 2 (éd. JACOBY).

${ }^{69}$ Sur le but politique de cette généalogie, qui soutient les revendications que, à l'époque de Phérécyde, les Philaides avançaient sur Salamine, l'île de leur ancêtre Ajax, $c f$. THomas (1989), p. 161-173; ЈАСОВ (1994), p. 190.
} 


\subsection{Hellanicos de Lesbos}

Hellanicos de Lesbos ${ }^{70}$, à la différence d'Acousilaos, Hécatée et Phérécyde, n'a pas composé une œuvre généalogique unique en plusieurs livres, mais une vaste série de syggraphai dont une partie est consacrée à des généalogies. Il semble ${ }^{71}$ que les Troica concluaient ce groupe d'écrits généalogiques tout en reprenant la plupart des gene présentés respectivement dans les Deukalioneia, la Phoronis, l'Asopis et l'Atlantis ${ }^{72}$. Il est alors probable que cet auteur ait pensé ses œuvres généalogiques comme un ensemble conclu par le contenu des Troica, à savoir par la guerre de Troie et les retours ${ }^{73}$. Il semble donc qu'une fois encore le temps généalogique raconté se termine avec la guerre de Troie et l'événement strictement lié qui est constitué par les nostoi. Ce plan diachronique général pourrait, toutefois, ne pas empêcher la présence de quelques généalogies qui se déroulaient au-delà de la guerre troyenne. Il est, en effet, possible, comme on l'a récemment soutenu ${ }^{74}$, que dans les Deucalioneia était présentée la généalogie de Codros, qui avait été roi d'Athènes après le conflit de Troie ${ }^{75}$. Le témoignage ${ }^{76}$ sur lequel on peut se fonder est une scolie qui semble dériver de deux œuvres d'Hellanicos : les Deucalioneia et l'Atthis. À la première le scoliaste aurait justement emprunté le long arbre généalogique de Codros, tandis que de la deuxième il aurait tiré le récit qui concerne la mort de ce personnage et la mention de son fils Nélée en tant que fondateur de la Dodécapole ionienne en Asie Mineure.

D'autre part, dans l'Asopis ${ }^{77}$ aussi, le temps raconté, avec un détour généalogique par les Philaides comparable à celui mis en scène par Phérécyde,

${ }^{70}$ Tandis qu'il semble certain qu'Hellanicos a vécu jusqu'aux dernières années de la guerre du Péloponnèse, dès l'antiquité sa date de naissance est incertaine, pour laquelle on a proposé la fin du $\mathrm{VI}^{\mathrm{e}}$ siècle av. J.-C., le début du $\mathrm{v}^{\mathrm{e}}$ ou la deuxième guerre médique : $c f$. récemment FowLER (2000), p. 147, et LENDLE (1992), p. 63-64.

${ }^{71}$ Cf. JACOBY (1957²b), p. 440 ad 4 F 23-31; vON FRITZ (1967), p. 480; LENDLE (1992), p. 65-66.

${ }^{72}$ En ce qui concerne, au contraire, le début du temps narré dans les œuvres généalogiques d'Hellanicos, il faut signaler que ce logographe, à la différence d'Acousilaos, ne racontait pas de théogonies, mais commençait sa narration par des «souches » hérö̈ques, à savoir, respectivement, Deucalion, Phoronée, les filles d'Asopos et celles d'Atlas : cf. LENDLE (1992), p. 65.

${ }^{73}$ Cf. VON FRITZ (1967), p. 480. Il n'est pas certain qu'Hellanicos ait présenté la généalogie des Tantalides (Schol. [A] Hom., Iliade II $105=$ Hellanicos, fr. 157 [éd. Fowler] = 4 F 157 [éd. JACOBY]) dans les Troica : cf. JACOBY (1957²b), p. 469-470 ad 4 F 157.

${ }^{74}$ Cf. FOWLER (2000), p. 201.

75 En effet, comme l'a observé SAKELlariou (1958), p. 307-308, la présente généalogie situe Codros à la quatrième génération après la guerre de Troie.

${ }^{76}$ Cf. Fowler (2000), p. 201, à propos de Schol. (T+) P1., Banquet, 208d = Hell., fr. 125 (éd. FOWLER) $=4 \mathrm{~F} 125$ (éd. JACOBY). Au contraire, d'après JACOBY $\left(1957^{2} \mathrm{~b}\right.$ ), p. 464 ad $4 \mathrm{~F} 125$ (éd. JACOBY), la narration dériverait entièrement de l'Atthis, à l'instar d'Harpocr., V, 141 = Hell., fr. 48 (éd. FOWLER) $=4 \mathrm{~F} 48$ (éd. JACOBY), un autre fragment sur la migration grecque en Ionie. En ce qui concerne cette colonisation chez Hellanicos, cf. aussi SAKELlariou (1958), p. 307-308, qui observe qu'elle est placée par le logographe à la quatrième génération après la guerre troyenne.

${ }^{77}$ Marcell., Vie de Thucydide II-IV = Hell., fr. 22 (éd. FOwlER) = 4 F 22 (éd. JACOBY). 
dépassait le conflit troyen, en arrivant jusqu'à l'époque de Miltiade, le colonisateur de la Chersonèse.

Chez Hellanicos, le conflit troyen ne représente pas seulement l'événement avec qui se termine le temps hérö̈que raconté dans les œuvres généalogiques, mais la prise de la ville est aussi l'objet de réflexions chronologiques $^{78}$, sur lesquelles les logographes, dans leur autonomie critique, avaient souvent des positions différentes ${ }^{79}$.

\section{La Bibliothèque du Pseudo-Apollodore}

Comme nous avons pu l'observer de la façon la plus claire avec le catalogue final de la Théogonie, mais aussi avec d'autres passages hésiodiques, les Généalogies d'Acousilaos et les œuvres généalogiques d'Hellanicos, même dans la Bibliothèque $e^{80}$ du Ps.-Apollodore, le temps raconté se termine avec la guerre de Troie suivie par les retours, dont le dernier est celui d'Ulysse. Cela est bien attesté non seulement dans les deux épitomés ${ }^{81}$ qui nous résument la conclusion perdue de l'œuvre ${ }^{82}$, mais aussi dans un passage de Photius ${ }^{83}$. Dans celui-ci, le patriarche byzantin atteste avoir pu lire un exemplaire complet de la Bibliothèque, dont il précise le contenu :

${ }^{78}$ Clem. Al., Stromates I, 104, 1-2 = Hell., fr. 152a (éd. FOWLER) = 323a F 21b (éd. JACOBY). Pour une attribution de ce fragment à l'œuvre chronographique d'Hellanicos Les Prêtresses d'Hèra, cf. récemment FOwLER (2000), p. 211, qui expose la même hypothèse pour Tzétzès, Posthomériques, $770-780=152 \mathrm{~b}$ (éd. Fowler) $=4$ F 152 (éd. JACOBY) et PbuG $40=$ fr. 152A (éd. Fowler) = 4 F 201 bis (éd. JACOBY).

79 Cf. Clém. Al., Stromates I, 104, 1-2 = Hell., fr. 152a (éd. FOwler) = 323a F 21b (éd. JACOBY). PbuG $40=$ Hell., fr. 152A (éd. FOWLER) = 4 F 201 bis (éd. JACOBY) atteste un désaccord chronologique entre Acousilaos et Hellanicos, mais à cause de nombreuses lacunes du texte il est difficile de bien saisir l'objet du débat ancien.

${ }^{80}$ Comme on le sait, l'auteur de la Bibliothèque est souvent dénommé Ps.-Apollodore, puisque, dès l'importante étude de ROBERT (1873), p. 11-28, son identification avec Apollodore d'Athènes, le fameux grammairien du II ${ }^{\mathrm{e}}$ s. av. J.-C., apparaît fausse. Elle était déjà proposée aussi bien par la tradition manuscrite que par Photius. La vraie identité de l'auteur nous échappe et également problématique est la datation de l'œuvre que, toutefois, il semble préférable d'attribuer aux premiers siècles de notre ère. $C f$., à ce propos, PELLIzER (1993), p. 295, qui affirme que l'auteur de la Bibliothèque ne se situe pas avant le $\mathrm{I}^{\text {er }}$ ou le $\mathrm{II}^{\mathrm{e}} \mathrm{s}$. ap. J.-C., et CARRIÈrE, MAssonIE (1991), p. 9-12, notamment p. 11, qui proposent comme terminus post quem Pausanias et comme terminus ante quem Philostrate, tout en suggérant donc une datation qui oscille entre la fin du $\mathrm{II}^{\circ}$ et le premier tiers du III $^{\mathrm{e}}$ siècle ap. J-C. Pausanias, en effet, ne semble pas connaitre la Bibliothèque, tandis que Philostrate apparaîtrait comme le plus ancien des auteurs qui l'ont connue. $C f$. plus récemment SCARPI, CIANI (1996), p. XII-XIII, qui accueillent cette datation, tout en n'excluant pas une chronologie quelque peu antérieure (61 av. J.-C. comme terminus ante quem).

${ }^{81}$ Il s'agit de l'Epitoma Vaticana (E), - probablement rédigée, au XII siècle ap. J.-C., par Jean Tzetzès - qui nous est donnée par un codex du XIV siècle (Vaticanus gr. 950), et des Fragmenta Sabbaitica (S), qui sont conservés par un codex du XIII siècle (Sabbaiticus-Hierosolymitanus 366) et qui, seuls, nous ont transmis le résumé des aventures d'Ulysse jusqu' à sa mort, avec laquelle se terminait la Bibliothèque. Cf. Diller (1935), p. 304-305; CARrière, Massonie (1991), p. 18; récemment, SCARPI, CIANI (1996), p. XIV-XV.

${ }^{82}$ Épit. I, 1 - VII, 40.

83 142a, 39 - 142b, 5 (éd. HeNRY). 


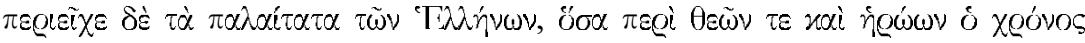

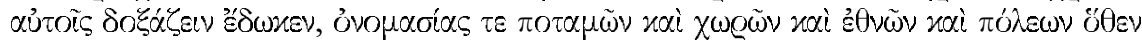

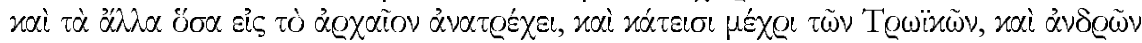

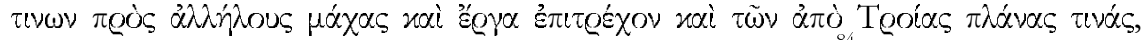
$\mu \dot{\alpha} \lambda \omega \sigma \tau \alpha$ \&' 'O

Le Ps.-Apollodore donc, en conduisant sa narration jusqu'aux errances d'Ulysse, accueille une donnée de la tradition. On observe, néanmoins, que, dans la section de la Bibliothèque consacrée aux événements troyens et aux retours $^{85}$, le récit est presque exclusivement diégétique, plutôt que généalogi$q^{8} e^{86}$ comme dans la conclusion de la Théogonie ou dans les syggraphai des logographes.

En effet, dans cette partie transmise par les épitomés, l'auteur semble avoir emprunté son sujet ni au Catalogue des femmes, sa source structurelle la plus importante $^{87}$, ni à d'autres ouvres généalogiques, mais aux poèmes homériques et au cycle troyen.

Le Ps.-Apollodore suit donc une tradition qui remonte au moins à l'époque archaïque. Toutefois il ne semble pas le faire d'abord pour un intérêt généalogique $^{88}$ ni, comme l'aède du Catalogue des femmes, pour réfléchir sur la nécessité de la disparition des demi-dieux. Il semble surtout soucieux de conserver $^{89}$ la tradition narrative grecque telle qu'elle est transmise par les

84 «Il (scil. : le petit livre) contenait les plus anciens événements des Grecs, et ce que le temps leur a donné à croire sur les dieux et sur les héros, les noms des fleuves, des pays, des peuples et des villes, d'où les autres faits qui remontent à l'époque ancienne, et il descend jusqu'aux événements de Troie, en traitant et les batailles de certains hommes les uns contre les autres et leurs exploits et quelques errances de ceux qui sont revenus de Troie, surtout celles d'Ulysse auquel le récit sur l'époque ancienne s'arrête. »

${ }^{85}$ Épit. 3, 1- 7, 40 .

${ }^{86}$ En même temps il faut, évidemment, signaler que la structure générale de l'œuvre est essentiellement généalogique. En ce qui concerne, en particulier, l’ordre des généalogies, $c f$. CARRIÈre (1998), p. 49, qui souligne que, dans la Biblothèque, on privilégie l'attention à la synchronie des générations héroïques, tandis que, dans le Catalogue desfemmes, on se concentre plutôt sur un déroulement diachronique très détaillé.

${ }^{87}$ Il s'agit d'une hypothèse largement reçue parmi les savants : cf. CASANOVA (1973), p. 3-27; WEST (1985), p. 35 et 43-46; CARRIÈRE, MASSONIE (1991), p. 13; maintenant, HiRsCHBERgER (2004), p. 32. Sur les sources de la Bibliothèque, cf. récemment CARRIÈRE, MAssonie (1991), p. 12-17, lesquels soulignent l'accès direct de l'auteur aux œuvres littéraires qu'il emploie. Sur ce dernier problème les savants ne sont pas encore complètement d'accord, car il semble aussi possible que le Ps.-Apollodore, dans certaines sections de son œuvre, ait employé un manuel mythographique : $c f$., récemment, HuYs (1997), p. 346-347.

${ }^{88}$ Toutefois, cela n'exclut pas que le Ps.-Apollodore ait été également confronté aux revendications, encore présentes parmi les aristocrates des villes grecques, de leurs ancêtres hérö̈ques et divins : $c f$., à ce propos, MACTOUX (1989), p. 264.

89 MACTOUX (1989), p. 66-69, mais cf. aussi 55-63, dénomme «tendance agglutinante » cette volonté du Ps-Apollodore de juxtaposer, tout en les conservant, les traditions anciennes. 
œuvres littéraires, comme le montre bien l'épigramme citée par Photius ${ }^{90}$ qui aurait accompagné, tout en le présentant, l'écrit mythographique ${ }^{91}$ :

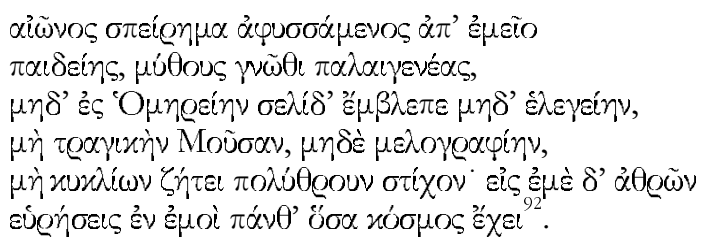

Les quelques lignes ${ }^{93}$ qui appartiennent à l'épitomé attestée par les fragmenta Sabbaitica et qui introduisent la section consacrée aux événements troyens nous semblent confirmer que la Bibliothèque est une œuvre récapitulative des narrations qu'on pouvait désormais lire dans les différentes productions littéraires :

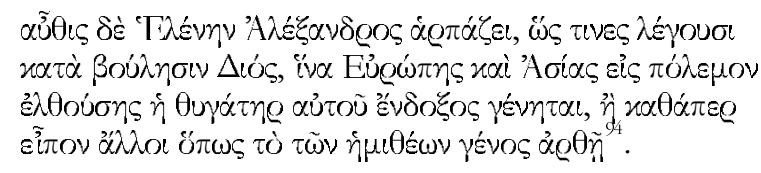

Le locuteur présente, sans choisir, deux causes de la guerre de Troie : la volonté, conçue par Zeus, de rendre célèbre Hélène ou bien de glorifier ${ }^{95}$ les demi-dieux. Il se borne donc à juxtaposer deux explications et à les placer, d'une façon traditionnelle, au commencement de sa narration troyenne.

En outre, on peut remarquer la manière dont, dans la suite de la narration telle qu'elle nous est transmise par les épitomés ${ }^{96}$, il n’y a plus de références au sort des demi-dieux, l'allusion initiale aux rimileot ayant seulement la

90 142b, 10-14 (éd. HeNRY).

${ }^{91}$ En effet, on a soutenu que cette épigramme pouvait être attribuée à l'auteur de la Bibliothèque: cf. DILLER (1935), p. 301; VAN DER VALK (1958), p. 167-168; plus récemment, CARRIÈRE, MASSONIE (1991), p. 163

92 «En puisant la spirale des âges dans mon érudution, connais les anciens récits, ne pose pas le regard sur la page homérique, ni sur l'élégie, ni sur la Muse tragique, ni sur la poésie mélique, ne recherche pas le très bruyant vers des poètes cycliques; en fixant les yeux sur moi, tu trouveras en moi tout ce que le monde contient. »

93 Ps.-Apoll., Bibliothèque, épit. 3, 1.

94 «Ensuite Alexandre enlève Hélène, comme certains le disent à cause de la volonté de Zeus, pour qu'à la suite de l'entrée en guerre d'Europe et d'Asie sa fille devienne célèbre, ou comme le dirent d'autres, afin que la génération des demi-dieux fût glorifiée. »

${ }^{95}$ Il nous semble, toutefois, que cet emploi de $\alpha$ 'c $\omega$ demeure ambigu, puisque ce verbe, au sens figuré, peut signifier autant «glorifier» (cf. TGL, s.v., I, 1045) qu' «éliminer, tuer» (cf. TGL, s.v., I, 1047). Même si les traductions concordent dans le choix du premier signifié ( $c f$. FRAZER [1921], p. 171; CARrière, MASSONIE [1991], p. 127; SCARPI, CiAni [1996], p. 327), qui, en effet, est plus fréquent, néanmoins, tout en considérant que, dans le corpus hésiodique, est racontée la fin des demi-dieux, on ne peut pas exclure que, dans le présent passage, a'ó signifie «éliminer ». Il s'agit d'une interprétation déjà proposée par BETHE (1891), p. 609, tandis que, la même année, WAGNER (1891), p. 396-397, montrait une préférence pour le signifié de «glorifier», tout en n'excluant pas l'autre.

${ }^{96}$ Ps.-Apoll., Bibliothèque, épit. 3, 2 - 7, 40. 
finalité de recueillir des données traditionnelles, à l'écart de toute importance dans la structure de l'œuvre.

\section{Conclusions}

Dans les œuvres considérées, le temps héroïque raconté se termine très souvent par la guerre troyenne, laquelle, excepté dans les Travaux, est immédiatement suivie par les retours et par l'indication des fils nés des participants au conflit.

Dans les narrations du corpus hésiodique, la fin de l'âge héroïque est considérée en tant que conclusion de l'époque des unions divino-humaines, comme nous le montre le catalogue final de la Théogonie, et en tant que disparition des mortels issus de ces amours, les demi-dieux, comme en témoignent le récit bien connu des Travaux et le fr. 204 M.-W. du Catalogue des femmes. De ce point de vue, le temps hérö̈que est donc une période d'intimité particulière entre les dieux et les hommes, dont les fruits sont les héros considérés, dans une perspective généalogique, comme demi-dieux. Comme il est bien attesté également dans l'Hymne à Aphrodite, la fin de cette période signe, alors, la conclusion d'un rapport spécial entre les sphères divine et humaine et le commencement d'un temps doué d'une qualité différente.

En ce qui concerne les logographes, Acousilaos considère de la manière la plus évidente la guerre de Troie, suivie par les retours, comme l'événement qui conclut le temps hérö̈que raconté, tandis qu'on n'est pas en mesure d'établir avec certitude si chez Hécatée aussi l'âge des héros se concluait avec les Troica ou bien si la narration concernait même un événement successif tel que la colonisation grecque en Ionie. Cet événement migratoire est connu par Hellanicos qui, néanmoins, dans le corpus généalogique de ses suggraphai, garde le conflit troyen comme limite temporelle de l'âge héroïque raconté. Chez Phérécyde, au contraire, la génération troyenne est certainement dépassée par celle de la migration ionienne en Asie Mineure.

Il est aussi important d'observer que, même quand les Troica sont le dernier événement narré, ces généalogistes en prose ne se concentrent pas sur la fin de l'intimité divino-humaine, mais préfèrent centrer leur attention sur la succession des générations dans les différentes lignées hérö̈ques. Celles-ci avaient déjà été traitées dans le Catalogue des femmes, mais sans perdre de vue la question du rapport entre le niveau des immortels et celui des mortels.

Dans la Bibliothèque du Ps.-Apollodore, on trouve une référence aux demi-dieux dans l'introduction à la partie consacrée aux Troica, avec lesquels se termine le temps héroïque raconté. Mais il ne s'agit que d'une mention synthétique sans effet sur le fil de la narration. En effet, l'auteur de la Bibliothèque souhaite garder les traditions anciennes, lesquelles, contrairement au traitement logographique, sont juxtaposées sans choix critique. Par rapport aux logographes, chez le Ps.-Apollodore les généalogies héroïques perdent leur importance spécifique pour accorder une plus grande attention aux récits 
des événements. La narration garde une structure diachronique fondée sur les généalogies, mais le contenu est très remarquablement centré sur l'intrigue.

Giovanni TOSETTI

Via Carrobbio, 17

I - 46010 S. MARTINO DALL'ARGINE (Mantova)

Courriel : gtosetti@libero.it

\section{Bibliographie}

G. ARRIGHETTI, Esiodo. Opere, Torino, Einaudi/Gallimard, 1998 (Biblioteca della Pléiade).

L. BerTelli, «Hecataeus: from Genealogy to Historiography », in N. LuRAGHI (éd.), The Historian's Craft in the Age of Herodotus, Oxford, Oxford University Press, 2001, p. 67-94.

E. BETHE, « Proklos und der epische Cyclus », Hermes 26 (1891), p. 593-633.

U. BIANCHI, Saggi di metodologia della storia delle religioni, Roma, Edizioni dell'Ateneo, 1979 (Nuovi Saggi)

C. CALAME, Thésée et l'imaginaire athénien. Légende et culte en Grèce antique, Lausanne, Payot, $1996^{2}$ [1990] (Sciences humaines).

—, «Feste, riti e forme poetiche », in S. SETTIS (éd.), I Greci. Storia, cultura, arte, società. II/1. Una storia greca. Formazione, Torino, Einaudi,1996a, p. 471-496.

-, Mythe et histoire dans l'Antiquité grecque. La création symbolique d'une colonie, Lausanne Payot, 1996b (Sciences humaines).

—, "Mémoire collective et temporalités en contact: Somare et Hérodote », RHR 215 (1998), p. 341-367.

- " "Tempo del racconto e tempo del rito nella poesia greca: Bacchilide tra mito, storia e culto », QUCC 62 (1999), p. 63-83.

—, Poétique des mythes dans la Grèce antique, Paris, Hachette, 2000 (Supérieur).

—, "Le funzioni di un racconto genealogico: Acusilao di Argo e la nascita della storiografia ", in P. Angeli-Bernardini (éd.), La città di Argo. Mito, storia, tradizioni poetiche. Atti del Convegno Internazionale (Urbino, 13-15 giugno 2002), Roma, Edizioni dell'Ateneo, 2004a, p. 229243 .

—, «Succession des ages et pragmatique poétique de la justice : le récit hésiodique des cinq espèces humaines », Kernos 17 (2004b), p. 67-102.

J.-C. CARrière, « Du mythe à l'histoire. Généalogies héroïques, chronologies légendaires et historicisation des mythes », in D. AUGER, S. SAÏD (éds), Généalogies mythiques. Actes du VIIIe Colloque du Centre de Recherches Mythologiques de l'Université de Paris-X (Chantilly, 14-16 septembre 1995), Paris, Les Belles Lettres, 1998 (Publications du Centre de recherches mythologiques de l'Université Paris X), p. 47-85.

J.-C. CARrière, B. MASsonie, La Bibliothèque d'Apollodore, Paris, Les Belles Lettres, 1991 (Lire les polyteismes).

A. CaSAnova, « Un frammento trascurato e il problema della divisione in libri del Catalogo esiodeo », SIFC 45 (1973), p. 3-27.

M.V. CerutTi, « Mito di distruzione, mito di fondazione. Hes. fr. 204, 95-103 M.-W. », Aevum Antiquum 11 (1998), p. 127-178.

J.S. ClaY, The Politics of Olympus. Form and Meaning in the Major Homeric Hymns, Princeton, New Jersey, Princeton University Press, 1989.

—, Hesiod's Cosmos, Cambridge, Cambridge University Press, 2003.

M. Crubellier, « Le mythe comme discours. Le récit des cinq races humaines dans les Travaux et les Jours », in F. Blaise, P. Judet DE LA COMBe, Ph. Rousseau (éds), Le métier du mythe. 
Lectures d'Hésiode, Lille, Presses Universitaires du Septentrion, 1996 (Cahiers de philologie), p. $431-463$

M. DAviES, The Epic Cycle, Bristol, Bristol Classical Press, 1989.

A. Diller, «The Text History of the Bibliotheca of Pseudo-Apollodorus», TAPhA 66 (1935), p. 296313.

P. DRÄGER, Untersuchungen zu den Frauenkatalogen Hesiods, Stuttgart, Steiner, 1997 (Palingenesia).

R.L. FOWLER, Early Greek Mythography, I, Oxford/New York, University Press, 2000.

J.G. Frazer, Apollodorus. The Library, London / New York, The Loeb Classical Library, 1921.

C.B. HASE, G.R.L. DE Sinner, T. FIX, Thesaurus Graecae Linguae, ab H. Stephano constructus, IVIII, Graz, Akademische Druck- und Verlagsanstalt, 1954, réimpr., [1831-1865].

M. HirschberGER, Gynaikôn Katalogos und Megalai Êhoiai. Ein Kommentar zu den Fragmenten zweier hesiodeischer Epen, München/Leipzig, Saur, 2004 (Beiträge zur Altertumskunde).

A. HoEkstra, The Sub-Epic Stage of Formulaic Tradition. Studies in the Homeric Hymns to Apollo, to Aphrodite and to Demeter, Amsterdam, Royal Netherlands Academy of Arts and Sciences, 1969 (Verhandeligen der Koninklijke Nederlandse Akademie von Wetenschappen, Afd. Letterkunde, Nieuwe Reeks).

M. HuYs, « 125 Years of Scholarship on Apollodoros the mythographer: A Bibliographical Survey », AC 66 (1997), p. 319-351.

C. JACOB, «L'ordre généalogique entre le mythe et l'histoire », in M. DETIENNE (éd.), Transcrire les mythologies, Paris, A. Michel, 1994 (Bibliothèque Albin Michel. Idées), p. 169-202.

F. JACOBY, Die Fragmente der Griechischen Historiker. I/A. Genealogie und Mythographie. Vorrede. Text. Addenda. Konkordanz, Leiden, E.J. Brill, $1957^{2}$ a [1923].

-, Die Fragmente der Griechischen Historiker. I/a. Genealogie und Mythographie. Komentar. Nachträge, Leiden, E.J. Brill, $1957^{2}$ b [1923].

R. JANKo, Homer, Hesiod and the Hymns. Diachronic Development in Epic Diction, Cambridge, University Press, 1982.

H. KLING, Griechische Papyrusurkunden aus ptolemäischer und römischer Zeit, Giessen, Töpelmann, 1924 (Mitteilungen aus der Papyrussammlung der Giessener Universitätsbibliothek).

O. LENDLE, Einführung in die griechische Geschichtsschreibung: von Hekataios bis Zosimos, Darmstadt, Wissenschaftliche Buchgesellschaft, 1992 (Die Altertumswissenschaft).

P. LÉvÊQuE, Bêtes, dieux et hommes. L'imaginaire des premières religions, Paris, Messidor, 1985 ( $L a$ passion de l'bistoire).

N. LuRAGHI, "Appunti sulla Ionia nella Geografia di Strabone », in A.M. BIRASCHI, G. SALMIERI (éds), Strabone e l'Asia Minore, Incontri Perugini di Storia della storiografia antica e sul mondo antico, X, Centro Studi Villa "La Colombella" (Perugia, 25-28 maggio 1997), Napoli, Edizioni Scientifiche Italiane, 2000 (Pubblicazioni dell'Università degli Studi di Perugia), p. 357-369.

M.-M. Mactoux, «Panthéon et discours mythologique. Le cas d'Apollodore », RHR 206 (1989), p. $245-270$.

J.R. MARCH, The Creative Poet. Studies on the Treatement of Myths in Greek Poetry, London, Institute of Classical Studies, 1987 (BICS, Suppl. 49).

R. Merkelbach, «Das Prooemium des hesiodeischen Katalogs », ZPE 3 (1968), p. 126-133.

R. Merkelbach, M.L. West, Fragmenta Hesiodea, Oxford, Clarendon Press, 1967.

O. MurRAY, « La Grecia degli 'eroi': mito, storia, archeologia », in S. SETTIS (éd.), I Greci. Storia, cultura, arte, società. II/1. Una storia greca. Formazione, Torino, Einaudi, 1996, p. 173-188.

E. Pellizer, « La mitografia », in G. CAMBIANO, L. CANFORA, D. LANZA (éds), lo spazio letterario della Grecia antica. I/2. La produzione e la circolazione del testo, Roma, Salerno Editrice, 1993, p. 283-303. 
C. ROBERT, De Apollodori Bibliotheca, dissert., Berlin, G. Schade, 1873.

A. RzAch, Hesiodi Carmina, Leipzig, Teubner, 1908² [1902] (Bibliotbeca Scriptorum Graecorum et Romanorum Teubneriana).

M.B. SAKellariou, La migration grecque en Ionie, Athènes, Institut français d'Athènes, 1958 (Collection de l'Institut français d'Athènes).

P. SCARPI, M.G. Ciani, Apollodoro. I miti greci (Biblioteca), Milano, Fondazione Lorenzo Valla, 1996.

F. Solmsen, Hesiodi Teogonia, Opera et Dies, Scutum. Fragmenta Selecta ediderunt R. MERKELBACH, M.L. WEST, Oxford, Clarendon Press, $1990^{3}$ (Scriptorum Classicorum Bibliotheca Oxoniensis).

R. Thomas, Oral Tradition and Written Record in Classical Athens, Cambridge, Univ. Press, 1989 (Cambridge Studies in Oral and Literate Culture).

M. VAN DER VALK, «On Apollodori Bibliotheca », REG 71 (1958), p. 100-168.

K. VON FRITZ, Die griechische Geschichtsschreibung. I. Von den Anfang bis Thukydides, Berlin, de Gruyter, 1967.

R. WAGNER, «Die Sabbaitischen Apollodorfragmente », RhM 46 (1891), p. 378-419.

M.L. West, Theogony, Oxford, Clarendon Press, 1966.

-, The Hesiodic Catalogue of Women. Its Nature, Structure and Origins, Oxford, Clarendon Press, 1985. 